3 Girard N, Vasiljevic A, Cottin V, et al. Respiratory failure with diffuse bronchiectases and cryoglobulinaemia. Eur Respir J 2008; 31: 1374-1378.

4 Khoor A, Myers JL, Tazelaar HD, et al. Amyloid-like pulmonary nodules, including localized light-chain deposition: clinicopathologic analysis of three cases. Am J Clin Pathol 2004; 121: 200-204.

5 Luraine R, Sohier L, Kerjouan M, et al. Une cause rare de maladie kystique pulmonaire : maladie à dépôts de chaînes légères d'immunoglobuline [An unusual cause of cystic lung disease: light chain deposition disease]. Rev Mal Respir 2013; 30: 567-571.

6 Colombat M, Mal H, Copie-Bergman C, et al. Primary cystic lung light chain deposition disease: a clinicopathologic entity derived from unmuted B cells with a stereotyped IGHV4-34/IGKV1 receptor. Blood 2008; 112: $2004-2012$.

7 Abraham RS, Geyer SM, Price-Troska TL, et al. Immunoglobulin light chain variable (V) region genes influence clinical presentation and outcome in light chain-associated amyloidosis (AL). Blood 2003; 101: 3801-3808.

8 Guidelines Working Group of UK Myeloma Forum, British Commitee for Standards in Haematology, British Society for Haematology. Guidelines on the diagnosis and management of AL amyloidosis. Br J Haematol 2004; 125: 681-700.

9 Dispenzieri A, Kyle RA, Lacy MQ, et al. Superior survival in primary systemic amyloidosis patients undergoing peripheral blood stem cell transplantation: a case-control study. Blood 2004; 103: 3960-3963.

10 Dimopoulos MA, Gertz MA, Kastritis E, et al. Update on treatment recommendations from the Fourth International Workshop on Waldenstrom's macroglobulinemia. J Clin Oncol 2009; 27: 120-126.

11 Gertz MA, Comenzo R, Falk RH, et al. Definition of organ involvement and treatment response in immunoglobulin light chain amyloidosis $(\mathrm{AL})$ : a consensus opinion from the 10th International Symposium on Amyloid and Amyloidosis, Tours, France, 18-22 April 2004. Am J Hematol 2005; 79: 319-328.

12 Dispenzieri A, Lacy MQ, Katzmann JA, et al. Absolute values of immunoglobulin free light chains are prognostic in patients with primary systemic amyloidosis undergoing peripheral blood stem cell transplantation. Blood 2006; 107: 3378-3383.

13 Leung N, Lager DJ, Gertz MA, et al. Long-term outcome of renal transplantation in light-chain deposition disease. Am J Kidney Dis 2004; 43: 147-153.

\title{
Reversal of central sleep apnoea with change from methadone to buprenorphine-naloxone: a case report
}

\section{To the Editor:}

Preventing prescription opioid poisoning deaths is a major public health priority in Western societies. Deaths from these medications exceed deaths from all illicit drugs combined [1]. Methadone (for pain treatment) is involved in one third of US prescription opioid overdose deaths despite accounting for only $5 \%$ of dispensed opioids [2]. There is a dose-dependent increase in the severity of central sleep apnoea (CSA) with methadone [3-5] and sleep disordered breathing is a contributing factor in methadone-related deaths [2]. The partial $\mu$-agonist buprenorphine is putatively safer than methadone with a ceiling effect upon respiratory depression [6]. However, the effect of buprenorphine on breathing during sleep remains unclear. The only relevant report from a cross-sectional observational study suggested that buprenorphine-naloxone therapy may induce significant CSA and hypoxaemia [7].

In contrast, we demonstrate a case of significant CSA reversed following a change from methadone to buprenorphine-naloxone therapy, together with improved hypoxaemia and normalised awake ventilatory control. A 47-year-old man who had been receiving methadone treatment for 3 years and with a 15-year of intravenous heroin use history was referred for overnight polysomnography (PSG). The patient had symptoms of snoring, witnessed sleep apnoeas and daytime sleepiness with an Epworth Sleepiness Scale (ESS) score of 11. During methadone treatment, he described frequent nausea, occasional vomiting, reduced libido and lack of motivation. He had no history of cardiac or neurological disease. His body mass index was $26 \mathrm{~kg} \cdot \mathrm{m}^{-2}$. He reported no recent substance use (confirmed by regular urinalysis over the preceding month) except from smoking $\sim 1 \mathrm{~g}$ cannabis per day. His oral methadone dose was $47.5 \mathrm{mg}$ once daily. Approximate peak plasma ( $2 \mathrm{~h}$ after dosing) [8] level of R-methadone was $184 \mathrm{ng} \cdot \mathrm{mL}^{-1}$ and $\mathrm{S}$-methadone was $213 \mathrm{ng} \cdot \mathrm{mL}^{-1}$. Olanzapine $10 \mathrm{mg}$ nocte was his only other medication, prescribed for anxiety by his general practitioner. His awake hypercapnic ventilatory response (HCVR) and hypoxic ventilatory response (HVR) was measured using a validated method [9]. 
After the first sleep study night, he switched from methadone to sublingual buprenorphine-naloxone as an outpatient, stabilising on once-daily $32 / 8 \mathrm{mg}$. After 10 weeks of buprenorphine-naloxone treatment, he had a follow-up PSG and ventilatory response test. He did not take other drugs excepting buprenorphine-naloxone, cannabis use (maintained at same quantity and frequency) and olanzapine $10 \mathrm{mg}$ nocte. Plasma concentrations $90 \mathrm{~min}$ after dosing (expected peak) [10] of buprenorphine was $11.4 \mathrm{ng} \cdot \mathrm{mL}^{-1}$ and of the metabolite norbuprenorphine was $15.8 \mathrm{ng} \cdot \mathrm{mL}^{-1}$. Comparison of the two PSGs and ventilatory control data is summarised in table 1.10 weeks after switching opioids, his CSA index dropped from 29.1 to 0.7 per h while his obstructive sleep apnoea/hypopnea index (OSAHI) remained 41 per h. His overnight oxygenation significantly improved and the change was not due to body supine position or rapid eye movement (REM) sleep (table 1). His HVR dropped by more than half, from 4.57 to $2.21 \mathrm{~L} \cdot \mathrm{min}^{-1} \cdot \mathrm{mmHg}^{-1}$. While on buprenorphine-naloxone, the patient noticed a reduction in witnessed apnoeas. His daytime sleepiness was improved, with an ESS score of 9. Previously reported symptoms of nausea and vomiting, reduced libido, and lack of motivation were also improved. He reported some experiences of constipation while on buprenorphine-naloxone.

Previous studies suggest that as many as $30 \%$ of stable methadone patients have CSA [3,5]. This case demonstrates that methadone-related CSA and oxygen desaturation during sleep can be resolved by changing to buprenorphine-naloxone therapy. The within-subject design suggests the findings are due to the change in prescribed opioid. The clinical effects of sublingual combination buprenorphine-naloxone formulation are generally attributable to buprenorphine alone as there is minimal sublingual naloxone absorption [10]. The patient transferred from $47.5 \mathrm{mg}$ oral methadone, with an oral morphine-equivalent (OME) dose of $223 \mathrm{mg}$, and stabilised on $32 \mathrm{mg}$ buprenorphine, equivalent to $1200 \mathrm{mg}$ OME [11], such that any changes are unlikely to reflect a lower total opioid dose.

In this case study, overall AHI is higher on methadone than on buprenorphine, while OSAHI is nearly identical. The only difference is the elimination of CSA events. Similar to our previous reported pattern, these long-term narcotic use-related CSA events are generally continuous, without concurrent sleep fragmentation, and unlikely to be related to REM sleep or supine position (table 1) [3, 5]. Methadone-related CSA rarely occurs in REM sleep, probably because the augmented HVR is naturally depressed during REM sleep [3, 12]. We found only one CSA event during REM sleep on methadone in this case. Therefore, REM-AHI was lower on methadone night, despite a higher overall AHI and \% supine REM sleep compared with the buprenorphine night.

It is possible that the elimination of CSA may due to the reduction of the augmented HVR. Our previous study has demonstrated that stable methadone users have significant CSA and a nearly doubled HVR [3, 12]. Similarly, augmented HVR is also a key mechanism of both high altitude-related CSA and heart failure-related CSA/Cheyne-Stokes respiration [13]. Increased loop gain, particularly controller gain (ventilatory chemosensitivity), has been identified to be a key mechanism of forming cyclical CSA events $[14,15]$. While acute opioid use is known to depress both HCVR and HVR, the increased HVR in chronic methadone users is probably a compensatory effect of the long-term depressed central respiratory controller/HCVR [12]. Indeed, our previous study showed that chronic methadone users had $29 \%$ lower

TABLE 1 Comparison of key PSG and ventilatory control parameters with methadone and buprenorphine therapy

Methadone

Buprenorphine

Central apnoea index per $h$
OSAHI per $h$
Overall AHI per $\mathrm{h}$
REM-AHI per $\mathrm{h}$
Total sleep time min
Supine sleep \%
Supine REM \%
Arousal index per $\mathrm{h}$
T90 min
ODI per h
HCVR L-min.
HVR L-1 $\cdot \mathrm{min}^{-1} \cdot \mathrm{mmHg}^{-1}$

29.1

41.1

70.2

44.7

440

84

10.4

23.2

39.9

60.3

2.10

4.57
0.7

41.2

41.9

57.5

527

94

4.3

9.1

15.2

34.0

1.51

2.21

OSAHI: obstructive sleep apnoea-hypopnoea index; $\mathrm{AHI}$ : apnoea-hypopnoea index; REM: rapid eye movement sleep; T90: sleep time with oxygen saturation measured by pulse oximetry $<90 \%$; ODI: index of oxygen desaturation $>3 \%$; HCVR: hypercapnic ventilatory response; HVR: hypoxic ventilatory response. 
HCVR and 91\% higher HVR compared with matched controls [12]. In our case study, with the elimination of the CSA by changing drugs, HVR dropped to half $\left(4.57\right.$ to $2.21 \mathrm{~L} \cdot \mathrm{min}^{-1} \cdot \mathrm{mmHg}^{-1}$ ) while HCVR also slightly decreased $\left(2.10\right.$ to $\left.1.51 \mathrm{~L} \cdot \mathrm{min}^{-1} \cdot \mathrm{mmHg}^{-1}\right)$. A reasonable explanation for the CSA improvement in our case would be likely through damping the augmented HVR rather than increasing HCVR.

Our findings contrast with a previous observational study, which suggested buprenorphine-naloxone may induce significant CSA and hypoxaemia [7]. PSGs on 70 opioid-dependent inpatients within 2 days of commencing buprenorphine-naloxone treatment found predominant CSA (average CSA index 11.4 per $\mathrm{h}$ ). Hypoxaemia, defined as an oxygen saturation measured by pulse oximetry $\left(\mathrm{SpO}_{2}\right)<90 \%$ for $>10 \%$ of sleep time, was present in $39 \%$ of patients [7]. However, PSG pre-initiation of buprenorphine was not measured, and patients may have had significant CSA and hypoxaemia with chronic opioid use pre-buprenorphine. Furthermore, the study was conducted at high altitude $\left(1500 \mathrm{~m}\right.$ ), where an $\mathrm{SpO}_{2}$ of $90 \%$ during sleep may be considered normal; indeed, the impact of the high altitude on CSA is also unclear [7].

Our report is limited by its single-case-study nature. We cannot easily explain the improvement in clinical symptoms from our findings. Nevertheless, our case study suggests that at least some methadone patients with CSA and/or hypoxia will have their symptoms resolved by switching to buprenorphine-naloxone. Given the alarming excess deaths with opioid use [16], there are significant clinical and public health benefits to confirming our observations and exploring relevant clinical outcomes in larger populations, preferably using randomised-controlled or within-subject designs.

- @ERSpublications

Methadone-using patients with central sleep apnoea may reverse symptoms with switch to buprenorphine-naloxone therapy http://ow.ly/NilUq

David Wang ${ }^{1,2,3,4}$, Nicholas Lintzeris ${ }^{4,5}$, Stefanie Leung ${ }^{4,5}$, Paul S. Haber ${ }^{4,5}$, Brendon J. Yee ${ }^{1,2,3,4}$ and Ronald R. Grunstein ${ }^{1,2,3,4}$

${ }^{1}$ Sleep and Circadian Group, Woolcock Institute of Medical Research, University of Sydney, Sydney, Australia. ${ }^{2}$ Department of Respiratory and Sleep Medicine, Royal Prince Alfred Hospital, Sydney Local Health District, Sydney, Australia. ${ }^{3}$ Centre for Integrated Research and Understanding of Sleep (CIRUS), University of Sydney, Sydney, Australia. ${ }^{4}$ Sydney Medical School, The University of Sydney, Sydney, Australia. ${ }^{5}$ Drug and Alcohol Services, South East Sydney Local Health District, Sydney, Australia.

Correspondence: David Wang, Woolcock Institute of Medical Research, 431 Glebe Point Road, Glebe NSW 2037, Australia. E-mail: david.wang@sydney.edu.au

Received: March 302015 | Accepted after revision: April 292015 | First published online: June 252015

Support statement: D. Wang is supported by an Australian National Health and Medical Research Council (NHMRC) Project Grant (1043633). R.R. Grunstein is supported by an NHMRC Practitioner Fellowship (1022730). Funding information for this article has been deposited with FundRef.

Conflict of interest: Disclosures can be found alongside the online version of this article at erj.ersjournals.com

\section{References}

1 Centers for Disease Control and Prevention. Addressing prescription drug abuse in the United States: current activities and future opportunities. www.cdc.gov/drugoverdose/pdf/hhs_prescription_drug_abuse_report_09.2013. pdf Date last updated: September 2013.

2 Webster LR, Cochella S, Dasgupta N, et al. An analysis of the root causes for opioid-related overdose deaths in the United States. Pain Med 2011; 12: Suppl. 2, S26-S35.

3 Wang $\mathrm{D}$, Teichtahl $\mathrm{H}$, Drummer $\mathrm{OH}$, et al. Central sleep apnea in stable methadone maintenance treatment patients. Chest 2005; 128: 1348-1356.

4 Walker JM, Farney RJ, Rhondeau SM, et al. Chronic opioid use is a risk factor for the development of central sleep apnea and ataxic breathing. J Clin Sleep Med 2007; 3: 455-461.

5 Wang D, Teichtahl H. Opioids, sleep architecture and sleep-disordered breathing. Sleep Med Rev 2007; 11: 35-46.

6 Dahan A, Yassen A, Romberg R, et al. Buprenorphine induces ceiling in respiratory depression but not in analgesia. Br J Anaesth 2006; 96: 627-632.

7 Farney RJ, McDonald AM, Boyle KM, et al. Sleep disordered breathing in patients receiving therapy with buprenorphine/naloxone. Eur Respir J 2013; 42: 394-403.

8 Eap CB, Buclin T, Baumann P. Interindividual variability of the clinical pharmacokinetics of methadone: implications for the treatment of opioid dependence. Clin Pharmacokinet 2002; 41: 1153-1193.

9 Wang D, Marshall NS, Duffin J, et al. Phenotyping interindividual variability in obstructive sleep apnoea response to temazepam using ventilatory chemoreflexes during wakefulness. J Sleep Res 2011; 20: 526-532.

10 Chiang CN, Hawks RL. Pharmacokinetics of the combination tablet of buprenorphine and naloxone. Drug Alcohol Depend 2003; 70: Suppl., S39-S47.

11 Nielsen S, Degenhardt L, Hoban B, et al. Comparing opioids: A guide to estimating oral morphine equivalents (OME) in research. Technical Report No. 329. Sydney, University of New South Wales, 2014.

12 Teichtahl H, Wang D, Cunnington D, et al. Ventilatory response to hypoxia and hypercapnia in stable methadone maintenance treatment patients. Chest 2005; 128: 1339-1347.

13 Dempsey JA, Veasey SC, Morgan BJ, et al. Pathophysiology of sleep apnea. Physiol Rev 2010; 90: 47-112. 
14 Dempsey JA, Xie A, Patz DS, et al. Physiology in medicine: obstructive sleep apnea pathogenesis and treatment considerations beyond airway anatomy. J Appl Physiol (1985) 2014; 116: 3-12.

15 White DP. Pathogenesis of obstructive and central sleep apnea. Am J Respir Crit Care Med 2005; 172: $1363-1370$.

16 Okie S. A flood of opioids, a rising tide of deaths. N Engl J Med 2010; 363: 1981-1985.

\title{
Linezolid tolerability in multidrug-resistant tuberculosis: a retrospective study
}

\author{
To the Editor:
}

Linezolid is used off-label to treat multidrug-resistant (MDR) and extensively drug-resistant (XDR) tuberculosis (TB) $[1,2]$. Recently, two systematic reviews and meta-analyses pointed out its promising efficacy $[3,4]$. However, linezolid toxicity may outweigh its potential benefits. Indeed, adverse events were notified in almost $60 \%$ of the treated cases, with a high incidence of severe events such as anaemia, peripheral neuropathy, optic neuritis, and thrombocytopenia. Decreased linezolid doses were associated with significantly lowered toxicity [5]. Furthermore, therapeutic drug monitoring (TDM) has increasingly been recognised as an asset in the field of TB treatment [6, 7]. TDM may assess individual linezolid exposure, especially since the drug shows a large inter-individual variability [8] and important pharmacological interactions were observed [6]. However, there is no clear association between linezolid exposure and adverse events. Drug exposure is not routinely evaluated, either in prospective studies or in routine care. Unfortunately, TDM is not routinely incorporated into the study designs of research upon MDR-TB and linezolid [9, 10]. Therefore, we aimed to retrospectively investigate linezolid safety and tolerability in relation to linezolid exposure in MDR-TB patients.

A retrospective study was conducted at two TB reference hospitals, the Tuberculosis Center Beatrixoord (University Medical Center Groningen, Haren, the Netherlands) and the Tuberculosis Reference Center for MDR-TB and HIV-TB E. Morelli Hospital (Sondalo, Italy). We selected patients that received linezolid as a part of their TB-treatment regimen [11]. Retrospective, consecutive data were retrieved by two researchers and consisted of: patient demographic, clinical, and epidemiological characteristics; drug susceptibility testing (DST) results; linezolid serum concentrations, reflected by an area under the time-concentration curve from 0-24 h (AUC0-24); and detailed information on the disease and treatment. If AUCs were available for different linezolid doses, the curve of the dose that was administered during the longest period of time was used for analysis. To assess safety and tolerability, data on adverse events, e.g. leukopenia, peripheral neuropathy, anaemia, thrombocytopenia, optic neuritis and gastrointestinal disorders, were collected from the hospital records or laboratory files using local reference values and procedures, representing clinical practice.

Of the original 112 patients 54 were excluded because they were: aged $<18$ years $(n=7)$, lacked data due to recent admission $(n=9)$, had drug-susceptible TB $(n=4)$, or their TDM data were unavailable $(n=34)$. Patients aged $<18$ years were excluded to rule out age-related developmental changes that might influence pharmacology. DST revealed susceptibility and resistance to a median (interquartile range (IQR)) number of $6.0(4.0-8.0)$ and $8.0(7.0-10.0)$ drugs) respectively. The median (IQR) for the number of different active drugs administered at any one time-point during treatment was 6.0 (5.0-7.0). DST revealed a median (IQR) minimum inhibitory concentration for linezolid of $0.5 \mathrm{mg} \cdot \mathrm{L}^{-1}\left(0.25-0.5 \mathrm{mg} \cdot \mathrm{L}^{-1}\right)$.

Table 1 describes the linezolid AUC0-24, cumulative dose, and number of days of exposure to linezolid for patients with and without linezolid-related adverse events. The distribution of AUC0-24 was the same for patients with and without anaemia $(\mathrm{p}=0.299)$, leukopenia $(\mathrm{p}=0.314)$, optical neuritis $(\mathrm{p}=0.612)$, and peripheral neuropathy $(\mathrm{p}=0.261)$. The distribution of minimum serum concentration ( $\mathrm{Cmin})$ of linezolid was the same across the categories anaemia $(p=0.169)$, leukopenia $(p=0.207)$, optical neuritis $(p=0.98)$, and peripheral neuropathy $(\mathrm{p}=0.477)$. Peripheral neuropathy was observed in $11(19 \%)$ patients. The distribution of linezolid cumulative dosage in $\mathrm{mg} \cdot \mathrm{kg}^{-1}(\mathrm{p}=0.041)$ and number of days of exposure to linezolid ( $\mathrm{p}=0.003$ ) were significantly higher in the patient group with peripheral neuropathy. Five $(9 \%)$ out of 45 patients (13 patients had missing data) had leukopenia: no differences were identified in cumulative linezolid dose or in days of exposure to linezolid ( $\mathrm{p}=0.194$ and 0.065 , respectively). Only one patient had optical neuropathy. 